\title{
Diagnostic Performance of F18-FDG PET/CT in Male Breast Cancers Patients
}

\author{
Andra Piciu ${ }^{1} \oplus$, Doina Piciu $2,3, * \oplus$, Narcis Polocoser ${ }^{1}$, Anita A. Kovendi ${ }^{1}$, Iulia Almasan ${ }^{2,3}$, Alexandru Mester ${ }^{4} \oplus$, \\ Dragos-Stefan Morariu ${ }^{5}$, Calin Cainap ${ }^{1} \mathbb{D}$ and Simona Sorana Cainap ${ }^{6}$
}

check for updates

Citation: Piciu, A.; Piciu, D.; Polocoser, N.; Kovendi, A.A.; Almasan, I.; Mester, A.; Morariu, D.-S.; Cainap, C.; Cainap, S.S. Diagnostic Performance of F18-FDG PET/CT in Male Breast Cancers Patients. Diagnostics 2021, 11, 119. https:/ / doi.org/10.3390/diagnostics 11010119

Received: 13 December 2020 Accepted: 11 January 2021 Published: 13 January 2021

Publisher's Note: MDPI stays neutral with regard to jurisdictional clai$\mathrm{ms}$ in published maps and institutional affiliations.

Copyright: (C) 2021 by the authors. Licensee MDPI, Basel, Switzerland. This article is an open access article distributed under the terms and conditions of the Creative Commons Attribution (CC BY) license (https:// creativecommons.org/licenses/by/ $4.0 /)$.
1 Department of Medical Oncology, Iuliu Hatieganu University of Medicine and Pharmacy, 400012 Cluj-Napoca, Romania; piciuandra@gmail.com (A.P.); narcispol@gmail.com (N.P.); kovendianita@yahoo.com (A.A.K.); calincainap2015@gmail.com (C.C.)

2 PhD School of Iuliu Hatieganu, University of Medicine and Pharmacy, 400012 Cluj-Napoca, Romania; almasan.iulia@gmail.com

3 Department of Endocrine Tumors and Nuclear Medicine, Institute of Oncology, 400012 Cluj-Napoca, Romania

4 Departement of Oral Health, University of Medicine and Pharmacy, 400012 Cluj-Napoca, Romania; alexandrumester@yahoo.com

5 Department of Surgery, University of Medicine and Pharmacy, 400012 Cluj-Napoca, Romania; dragosstefanmorariu@gmail.com

6 Department of Mother and Child, Iuliu Hatieganu University of Medicine and Pharmacy, 400012 Cluj-Napoca, Romania; simona.cainap@yahoo.com

* Correspondence: doina.piciu@gmail.com

\begin{abstract}
Introduction: F18-FDG PET/CT is the most important hybrid imaging used in the diagnostic, staging, follow-up, and treatment evaluation response in cancer patients. However, it is well-known that in breast cancer the use of F18-FDG is not included in the first line protocol of initial diagnostic, both in female and male breast cancer patients. F18-FDG PET/CT is a valuable tool to provide information on extra-axillary lymph node involvement, distant metastases, and other occult primary cancers. This study assesses F18-FDG PET/CT systemic staging in male patients with diagnosed breast cancer and determines detection rates for unsuspected distant metastases and synchronous malignancies. Methods: We analyzed a number of 170 male patients with breast cancer, seen between 2000-2020, in a tertiary center. From this group, between 2013-2020 a number of 23 patients underwent F18-FDG PET/CT. Rates of upstaging were determined for each case and the detection of other primary malignancies was analyzed. Results: Median age of male breast cancer group was $61.3 \mathrm{y}$ (range, 34-85 y), most had intraductal carcinoma (82.4\%) and unsuspected distant metastases, which increased patient stage to IV, observed in $27 \%$. In 4 out 23 patients (17.4\%), F18-FDG PET/CT identified synchronous cancers (2 prostate cancers, 1 thyroid and 1 colon cancer). Conclusion: F18-FDG PET/CT is a valuable tool to provide information on extra-axillary lymph node involvement, distant metastases, and other occult primary cancers. Baseline F18-FDG PET/CT has a substantial impact on the initial staging and on clinical management in male breast patients and should be considered for use in newly diagnosed patients.
\end{abstract}

Keywords: male breast cancer; synchronous malignancies; F18-FDG PET/CT

\section{Introduction}

The PET/CT hybrid imaging is an essential tool in primary diagnostic, staging, followup, and treatment response evaluation of many malignant diseases. Compared to other cancers, in breast cancer the use of F18-FDG PET/CT has proven clinical value in selected cases: in patients with newly diagnosed breast cancer, F18-FDG PET/CT is recommended for stage III disease [1] and for restaging, follow-up, and treatment response evaluation. Some studies recommend due to the detection of unsuspected distant metastases, fact that alters treatment and prognosis, that the guidelines should consider adding in patients with stage IIB breast cancer the systemic staging with F18-FDG-PET/CT at the time of the 
initial diagnosis [2-5]. F18-FDG PET/CT reduced false-positive risk by half and decreased workup of incidental findings, allowing earlier treatment and being cost-effective [4]. Approximately $37 \%$ of patients with clinical stage IIA-IIIC breast cancer who underwent F18-FDG PET/CT before preoperative systemic therapy showed more extensive disease, including $23 \%$ with more extensive nodal metastasis and $14 \%$ with distant metastasis [5]. The male breast cancer represents only $1 \%$ of all breast cancers $[3,6]$ and the literature is by far less consistent than in women, studies have limited number of subjects and many are case reports [6-11]. In a recent study published by Ulaner et al. [7] it is underlined that the role of F18-FDG PET/CT is based on female guidelines and that an analysis of the value of F18-FDG PET/CT for systemic staging of male breast cancer is warranted. In this study, we retrospectively search the database of a tertiary cancer center to evaluate the value of F18-FDG PET/CT in male breast cancer patients, in detecting the presence of synchronous tumors.

\section{Materials and Methods}

\subsection{Study Design}

This retrospective single-institution study was performed on a database of a Romanian tertiary cancer center, the Institute of Oncology "Prof. Dr. Ion Chiricuță" Cluj-Napoca (IOCN) searching for patients with male breast cancer between January 2000 and November 2020. The inclusion criteria were: male breast cancer histological confirmed; hormonal receptors status: estrogen receptor $(\mathrm{ER})$, progesterone receptor $(\mathrm{PR})$, and human epidermal growth factor receptor 2 (HER2) described; we found an important number of patients $(43 \%)$ without HER 2 status defined, because of the lack of determinations in the first decade of the database; all patients signed the institutional informed consent both for diagnostic and treatment procedure, and for the use of their data in scientific reports, with personal data protection respected. The study protocol was approved by the Ethics Committee of the Institute of Oncology "Prof.Dr.I.Chiricuta" Cluj-Napoca, approval number 174/28.02.2020. The study was conducted according to the principles of the Declaration of Helsinki, the International Conference on Harmonization Guideline on Good Clinical Practice, the Romanian laws and regulations.

Epidemiologic data on this cohort and the survival parameters are reported in this study. Between August 2013-November 2020 from this cohort 23 patients have been evaluated by F18-FDG PET/CT in different moments of the disease: staging, restaging, and during monitoring. In this group the hybrid imaging parameters were analyzed: presence or absence of pathologic F18-FDG uptake, number of lesions, standardized uptake values (SUV). All lesions suspected for synchronous primary tumors were registered and followed for their histological confirmation.

\subsection{PET/CT Imaging}

All hybrid images were obtained with a GE Optima 560 PET/CT, GE Healthcare USA; F18-FDG PET/CT studies were performed after a period of fasting of $6 \mathrm{~h}$; the blood glucose levels between $76 \mathrm{mg} / \mathrm{dL}-148 \mathrm{mg} / \mathrm{dL}$ were measured before the F18-FDG injection; injected doses had average activity of 241.4 MBq (Min 148-Max 380 MBq). F18-FDG activities were calculated according to the patients' weight and by the European Association of Nuclear Medicine (EANM) procedure guidelines for tumor imaging, version 2.0 [12] and the examination was performed after an uptake period of 50-70 min. CT images, with a slice thickness of $3.75 \mathrm{~mm}$, were acquired using a low-dose protocol $(100-130 \mathrm{kV}, 50-100$ auto $\mathrm{mA}$, index noise of $20 \%$ ) in order to reduce the irradiation dose for patients. F18-FDG $\mathrm{PET} / \mathrm{CT}$ images were evaluated by a nuclear medicine physician and a radiologist. For all F18-FDG PET/CT studies, SUVlbm (the standardized uptake value lean body mass) a semi quantitative parameter for F18-F18-FDG uptake calculation respecting a standard protocol on the work station (Volumetrix for PET/CT) was used. 


\subsection{Statistical Analysis}

Statistical analysis was performed using GraphPad Prism 6.0 software. We calculated means and standard deviations.

\section{Results}

A total of 170 male patients diagnosed with breast cancer from 2000-2020 were analyzed. In a period of 20 years, the male breast cancer represents $0.1 \%$ from all breast cancer reported in our institution. The mean $\pm \mathrm{SD}$ age was $61.34 \pm 11.19$ year-old. The majority, 140 patients (82.4\%) had the histology of intraductal carcinoma (IDC), 7 cases (5\%) had invasive lobular carcinoma (ILC), 4 cases (2.4\%) had in situ ductal carcinoma, and 19 cases (11.2) had other forms. Regarding the receptor immunohistochemistry, 10 patients (5.6\%) were ER negative, 138 cases ( $81.2 \%$ ) were ER positive, and in 22 patients $(12.9 \%)$ the ER status was not available. Twelve patients $(7 \%)$ were PR negative and $137(80.6 \%)$ were PR positive, in 21 patients (12.3\%) PR status was not available. HER2 was not available in 79 cases (46.5\%); was 0 in 43 cases (25.3\%); 1 in 26 cases $(15.3 \%)$; 2 in 15 cases $(8.8 \%)$; 3 in 7 cases (4.1\%). None of our patients was triple negative. At the moment of the report 95 patients were alive $(55.8 \%), 65$ patients $(38.2 \%)$ were dead, and in 10 cases the survival data were not available. Thirty-two patients $(60.3 \%)$ in stage III of the disease were alive at the moment of the study, treated by radical mastectomy and ALND, radiotherapy and chemotherapy; 8 patients with stage II and III presented metastatic recurrence of the disease during the first 2 years, but are alive. Among the 65 dead patients, 21 were in stage III, 5 patients were diagnosed in stage IV, and 39 of them had an unknown cause of the death, less likely related to their disease.

The main characteristics of the database is presented in Table 1.

Table 1. Characteristics of male patients with breast cancer in IOCN 2000-2020.

\begin{tabular}{cc}
\hline Age (Years) & $\mathbf{6 1 . 3 4}$ \\
\hline Tumor size & 4 \\
T in situ & 20 \\
T1 & 43 \\
T2 & 16 \\
T3 & 68 \\
T4 & 19 \\
NA & \\
Grading (G) & 35 \\
3 (High) & 92 \\
2(Intermediate) & 18 \\
1 (Low) & 35 \\
NA & \\
Lymph nodes (N) status & 13 \\
N1 & 48 \\
N2 & 33 \\
N3 & 15 \\
NA & 61 \\
Metastasis (M) & \\
M0 & 151 \\
NA & $6 / 5 / 4 / 1 / 1$ \\
Receptors status & 2 \\
ER+/ER-/NA $/$ PR-/NA & $138 / 10 / 22$ \\
HER2-0/1/2/3/NA & $43 / 26 / 15 / 79$ \\
\hline
\end{tabular}


Table 1. Cont.

\begin{tabular}{|c|c|}
\hline Age (Years) & 61.34 \\
\hline \multicolumn{2}{|l|}{ Stage } \\
\hline IA,B & 6 \\
\hline IIA & 17 \\
\hline IIB & 9 \\
\hline IIIA & 12 \\
\hline IIIB & 27 \\
\hline IIIC & 14 \\
\hline IV & 6 \\
\hline NA & 79 \\
\hline \multicolumn{2}{|l|}{ Surgical Therapy } \\
\hline Radical mastectomy & 149 \\
\hline Breast conservative surgery & 2 \\
\hline No surgery & 19 \\
\hline ALND & 19 \\
\hline SLND & 2 \\
\hline No axillary evaluation & 19 \\
\hline \multicolumn{2}{|l|}{ External Beam Radiotherapy } \\
\hline Yes & 74 \\
\hline No & 96 \\
\hline \multicolumn{2}{|l|}{ Neoadjuvant chemotherapy } \\
\hline Yes/No & $55 / 115$ \\
\hline \multicolumn{2}{|l|}{ Adjuvant chemotherapy } \\
\hline Yes/No & $62 / 118$ \\
\hline \multicolumn{2}{|l|}{ Adjuvant antiestrogenic therapy } \\
\hline Yes/No & $102 / 68$ \\
\hline Alive/Death/NA & $95 / 70 / 5$ \\
\hline
\end{tabular}

NA-not available data.

The analysis of this report revealed an unusual high number of patients with skin and subcutaneous metastases, 6 out of $17(35.3 \%)$ in the group of subjects with distant metastases, the majority being discovered on F18-FDG PET/CT scans, Figure 1.

From the above mentioned database, between 2013 and 2020, a number of 23 patients were evaluated by hybrid imaging F18-FDG PET/CT, Figure 2. The male breast cancers represented $2.06 \%$ from all scans performed for breast carcinomas. The percentage of scans is considerably higher $(2.06 \%)$ compared to the percent of male breast cancer among all breast carcinoma cases $(0.1 \%)$. A number of 4 patients $(17.4 \%)$ were discovered with multiple primary malignancies during the metabolic scan, confirmed in histology: 1thyroid cancer, 1 - prostate cancer, 1 -Hodgkin lymphoma, and 1-colon cancer. Table 2.

The characteristics of patients followed by F18-FDG PET/CT scan are presented in

The cohort of patients submitted for F18-FDG PET/CT scan has the mean age $60.69 \pm 9.216$ year-old, age slightly lower compared to the whole cohort of male patients with breast cancers.

F18-FDG PET/CT scans was performed for restaging in 5 cases (21.7\%), for the increase of tumor markers in $13(56.5 \%)$, assessment for response to therapy in $2(8.7 \%)$, and during follow-up in 3 cases (13\%). The scans demonstrated new, unknown lesions suggestive for metastases in 12 (52.2\%) out of 23 patients. The unsuspected metastatic sites included: skeleton, liver, sub-pectoral and mediastinal lymphnodes, lungs, muscles, subcutaneous, and skin. The rate of upstaging was $26 \%$, occurring in 6 patients. 


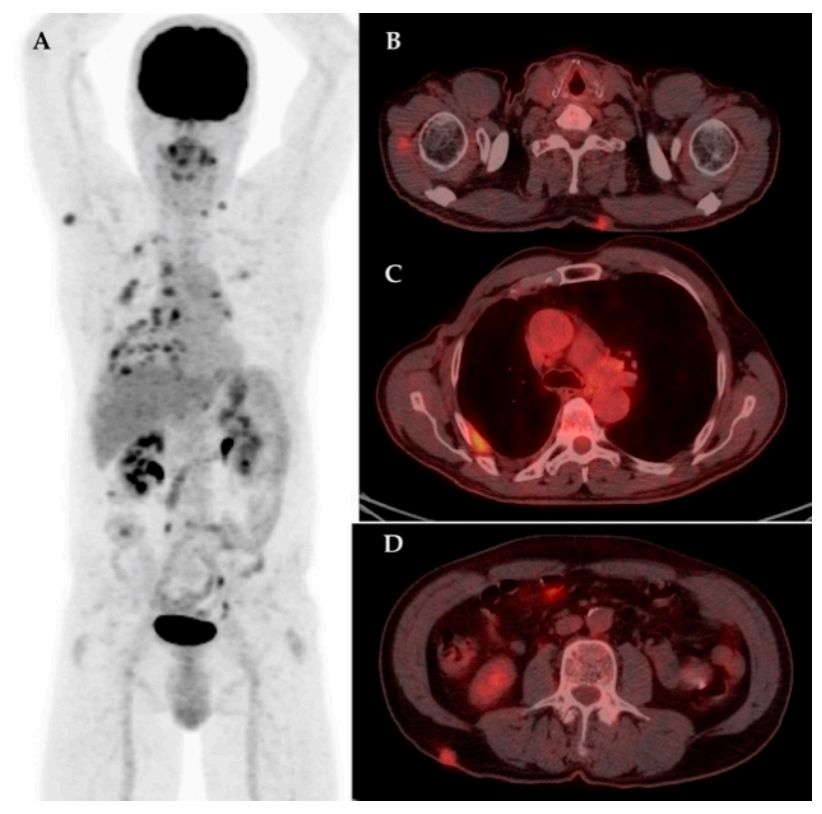

Figure 1. Male patient with right breast cancer, with right radical mastectomy and axillary lymphnode dissection, with multiple sites of increased F18-FDG uptake confirmed as breast carcinoma metastases. MIP image on F18-FDG PET/CT (A); axial section at cervical level showing muscle and posterior cervical subcutaneous lesions with increased F18-FDG uptake confirmed metastases on biopsy (B); axial section at thorax level showing pathologic increased F18-FDG uptake in the right pleura (C); axial section at lumbar level showing right posterior lumbar subcutaneous lesion with increased F18-FDG uptake confirmed metastases on biopsy (D).

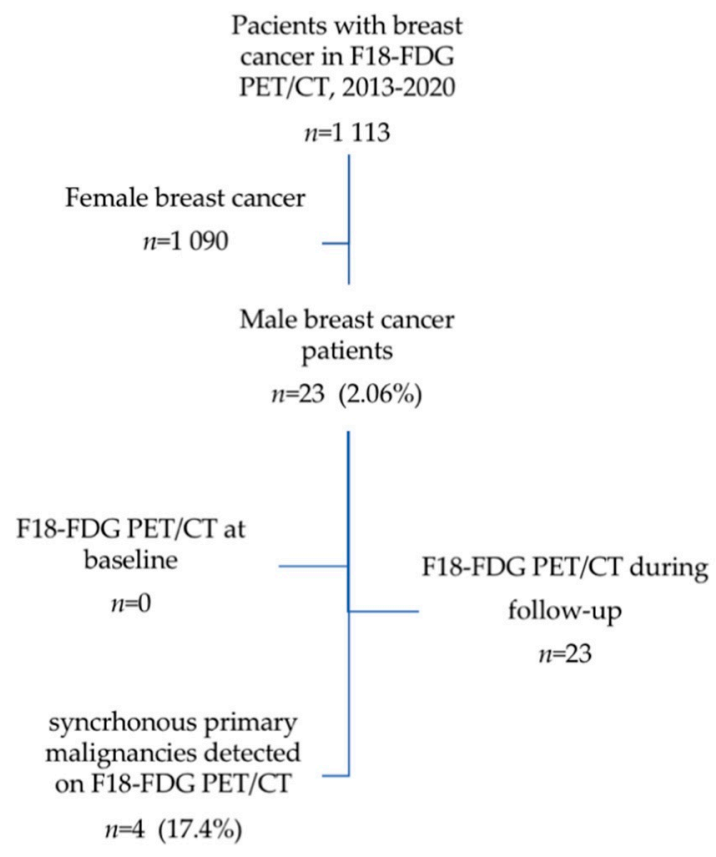

Figure 2. Male breast cancer patients evaluated by F18-FDG PET/CT 2013-2020. 
Table 2. The characteristics of patients followed by F18-FDG PET/CT scan.

\begin{tabular}{|c|c|c|c|c|c|c|c|c|c|c|c|}
\hline No. & Age & Histo. & Surgery & CHT & EBR & Stage & Mets * & $\begin{array}{c}\text { New } \\
\text { Mets ** }\end{array}$ & $\begin{array}{l}\text { SUV1 } \\
\text { Max }\end{array}$ & $\begin{array}{c}\text { Synchronous } \\
\text { Cancer }\end{array}$ & $\begin{array}{l}\text { SUV2 } \\
\text { Max }\end{array}$ \\
\hline 1 & 66 & IDC & RM + SLND & Yes & Yes & IV & $\mathrm{LN}, \mathrm{Bn}$ & $\mathrm{LN}, \mathrm{Lu}$ & 7.04 & - & - \\
\hline 2 & 60 & IDC & $\mathrm{RM}+\mathrm{ALND}$ & NA & Yes & IIIA & - & - & - & - & - \\
\hline 3 & 66 & IDC & $\mathrm{RM}+\mathrm{ALND}$ & - & - & IIA & - & - & - & - & - \\
\hline 4 & 61 & IDC & RM & - & - & IIA & - & - & - & - & - \\
\hline 5 & 70 & IDC & $\mathrm{RM}+\mathrm{ALND}$ & - & - & IB & - & $\mathrm{Lu}, \mathrm{Su}$ & 3.37 & Colon cancer & 5.27 \\
\hline 6 & 52 & IDC & $\mathrm{RM}+\mathrm{ALND}$ & - & - & IIA & - & LN & 4.77 & - & - \\
\hline 7 & 47 & IDC & Biopsy & - & Yes & IIIC & LN & $\mathrm{Bn}, \mathrm{Sk}$ & 18.36 & - & - \\
\hline 8 & 52 & IDC & $\mathrm{RM}+\mathrm{ALND}$ & Yes & Yes & IV & $\mathrm{LN}, \mathrm{Bn}$ & $\mathrm{Lu}$ & 4.38 & - & - \\
\hline 9 & 67 & IDC & $\mathrm{RM}+\mathrm{ALND}$ & Yes & Yes & IIA & - & - & - & - & - \\
\hline 10 & 57 & IDC & $\mathrm{RM}+\mathrm{ALND}$ & NA & NA & IB & - & LN & 1.55 & - & - \\
\hline 11 & 47 & IDC & $\mathrm{RM}+\mathrm{ALND}$ & - & - & IIB & - & $\mathrm{LN}, \mathrm{Bn}$ & 10.61 & - & - \\
\hline 12 & 79 & PBC & $\mathrm{RM}+\mathrm{ALND}$ & Yes & Yes & IB & - & - & - & - & - \\
\hline 13 & 67 & IDC & $\mathrm{RM}+\mathrm{ALND}$ & - & - & IIB & LN & LN, L & 4.43 & - & - \\
\hline 14 & 68 & IDC & $\mathrm{RM}+\mathrm{ALND}$ & Yes & Yes & IIIB & LN & $\mathrm{LN}, \mathrm{Bn}$ & 7.04 & $\begin{array}{l}\text { Prostate } \\
\text { cancer }\end{array}$ & $5.01-$ \\
\hline 15 & 52 & IDC & $\mathrm{RM}+\mathrm{ALND}$ & Yes & Yes & IIB & - & - & - & - & - \\
\hline 16 & 64 & ILC & $\mathrm{RM}+\mathrm{ALND}$ & - & - & IIA & - & - & - & - & - \\
\hline 17 & 61 & IDC & RM + ALND & Yes & Yes & IIIB & LN & $\begin{array}{l}\mathrm{LN}, \mathrm{Lu}, \\
\mathrm{M}, \mathrm{Bn}\end{array}$ & 3.1 & $\begin{array}{c}\text { Hodgkin } \\
\text { Lymphoma }\end{array}$ & 11.6 \\
\hline 18 & 42 & IDC & $\mathrm{RM}+\mathrm{ALND}$ & Yes & Yes & IIIC & - & - & - & - & - \\
\hline 19 & 74 & IDC & $\mathrm{RM}+\mathrm{ALND}$ & Yes & Yes & IIIC & $\mathrm{LN}, \mathrm{Bn}$ & $\begin{array}{c}\text { LN, Sk, } \\
\mathrm{M}\end{array}$ & 6.21 & - & - \\
\hline 20 & 59 & IDC & $\mathrm{RM}+\mathrm{ALND}$ & Yes & Yes & IIIB & - & LN, Sk & 8.6 & - & - \\
\hline 21 & 61 & IDC & RM + ALND & Yes & Yes & IIC & LN & - & 2.38 & $\begin{array}{l}\text { Thyroid } \\
\text { cancer }\end{array}$ & 23.1 \\
\hline 22 & 69 & IDC & $\mathrm{RM}+\mathrm{ALND}$ & NA & NA & IIA & - & - & - & - & - \\
\hline 23 & 55 & ILC & $\mathrm{RM}+\mathrm{ALND}$ & Yes & Yes & IIB & - & - & - & - & - \\
\hline
\end{tabular}

Histo-histology; IDC-ductal invasive carcinoma; ILC-invasive lobular carcinoma; PBC-papillary breast carcinoma; RM-radical mastectomy; ALND-axillary lymphnode dissection; SNL-sentinel lymphnode biopsy; CHT-chemotherapy; EBR-external beam therapy; Mets *-known metastases; New mets **-unsuspected metastases detected on F18-FDG PET/CT; LN-lymphnode; L-liver; Lu-lung; Skskin; Su-subcutaneous; Bn-bone; B-brain; M-muscle; SUV1Max-maximum standardized uptake value in the hottest known metastasis; SUV2Max-maximum standardized uptake value in the lesion suspected as synchronous primary cancer; NA-unavailable data.

The synchronous primary malignancies were demonstrated in 4 cases, confirmed in histology after: total thyroidectomy for papillary thyroid carcinoma; cervical lymphnode biopsy for Hodgkin lymphoma; prostatic biopsy for prostate cancer and left hemicolectomy for colon adenocarcinoma. In Figures 3-6 we present the images of patients with synchronous tumors detected in F18-FDG PET/CT scans during restaging, tumor marker increased, or follow-up. 

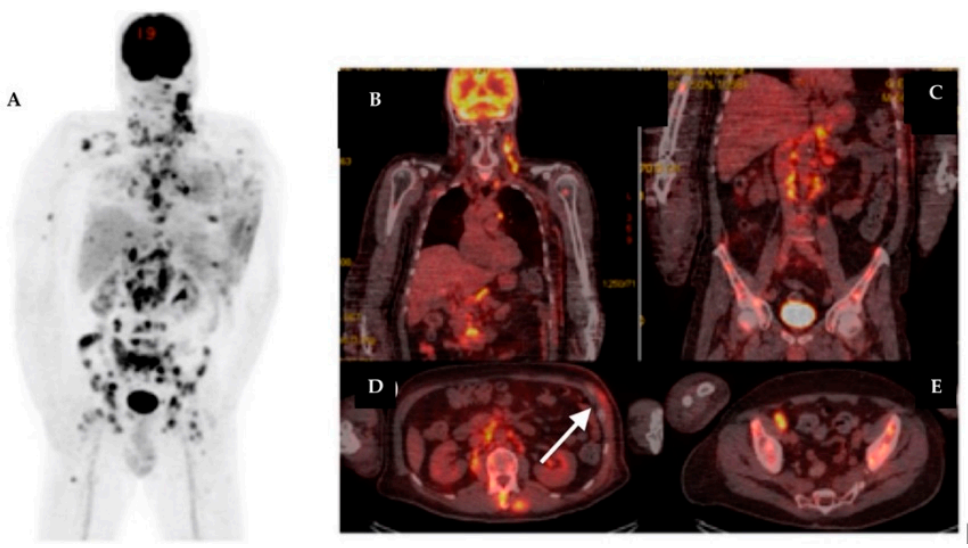

Figure 3. Male patient with left breast cancer and multiple metastatic lesions. Synchronous confirmed Hodgkin lymphoma. MIP image on F18-FDG PET/CT (A); coronal section at thorax level showing multiple cervical and mediastinal pathologic lymph nodes, with increased F18-FDG uptake (B); coronal section at abdominal level showing multiple pathologic lymph nodes with increased F18FDG uptake in retroperitoneal area and multiple bone lesions, confirmed as metastatic from breast cancer (C); axial section at upper abdominal level showing multiple pathologic lymph nodes with increased F18-FDG uptake in retroperitoneal area and multiple bone and muscle lesions (D) and infiltration of left breast area posttherapy, with recurrence of the tumor (white arrow); axial section at pelvic level showing pathologic lymph node with increased F18-FDG uptake in right external iliac area and multiple bone lesions (E).

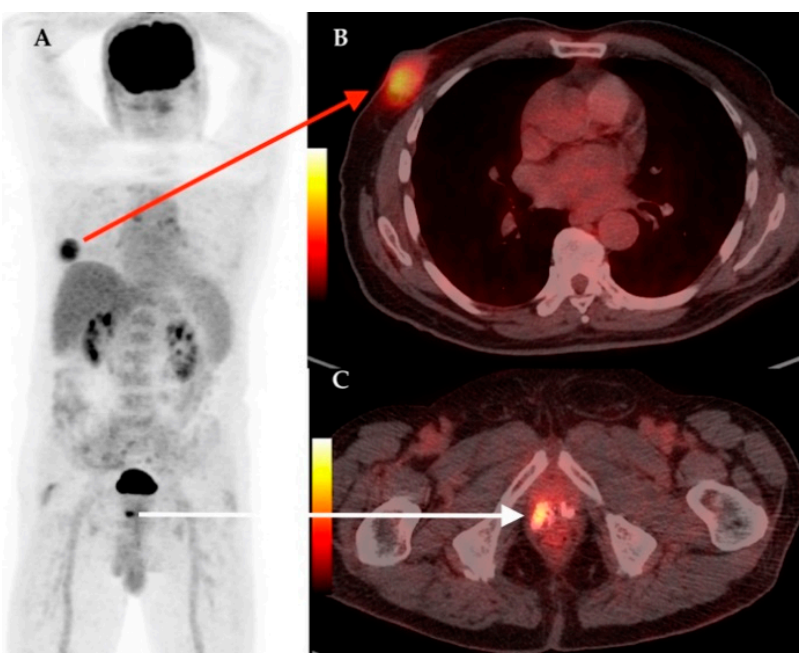

Figure 4. Male patient with right breast cancer. Synchronous confirmed prostate adenocarcinoma. MIP image on F18-FDG PET/CT (A); axial section at thorax level showing right breast lesion with increased F18-FDG uptake confirmed breast carcinoma on biopsy (B, red arrow); axial section at pelvic level showing pathologic increased F18-FDG uptake in right prostate lobe area with multiple calcification (C, white arrow), confirmed prostate adenocarcinoma. 


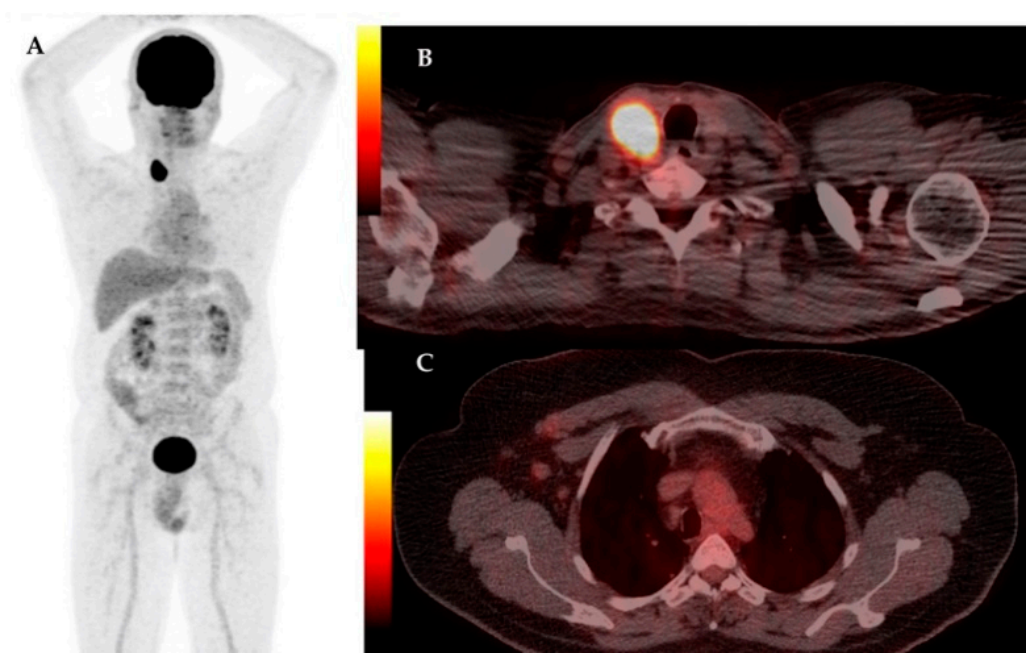

Figure 5. Male patient with right breast cancer, with right axillary lymphnodes suggestive for metastases. Synchronous confirmed papillary thyroid carcinoma of the right lobe. MIP image on F18-FDG PET/CT (A); axial section at cervical level showing thyroid right lobe lesion with increased F18-FDG uptake confirmed thyroid carcinoma on total thyroidectomy (B); axial section at thorax level showing pathologic slightly increased F18-FDG uptake in right axilla in the lymphnodes, confirmed metastatic (C).

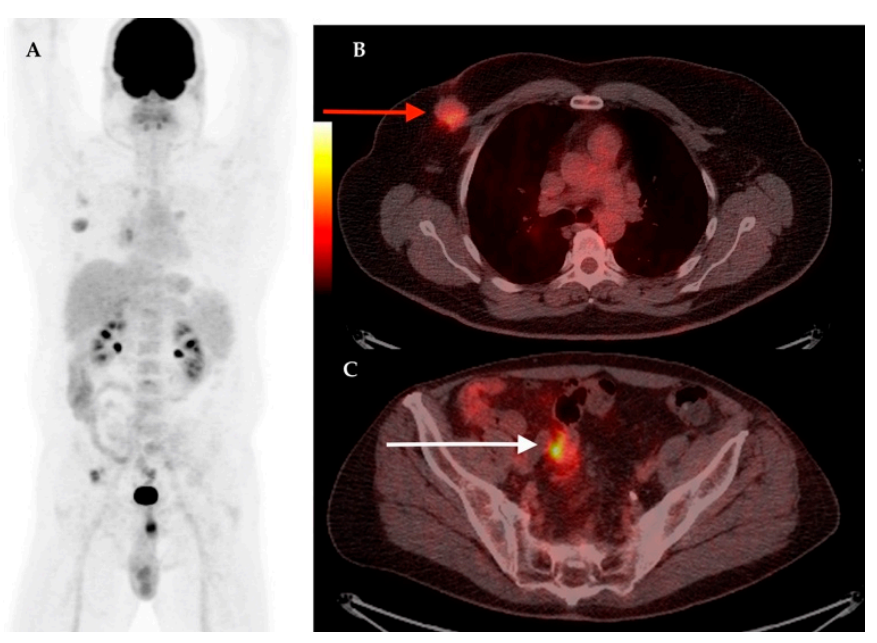

Figure 6. Male patient with right breast cancer. Synchronous confirmed colonic adenocarcinoma. MIP image on F18-FDG PET/CT (A); axial section at thorax level showing right breast lesion with increased F18-FDG uptake confirmed breast carcinoma on biopsy (B, red arrow); axial section at pelvic level showing pathologic increased F18-FDG uptake in the colon (C, white arrow), confirmed colon adenocarcinoma in biopsy at colonoscopy.

\section{Discussion}

In a period of 20 years, 2000-2020, we report a number of 170 cases of male breast cancer patients, representing $0.1 \%$ from all breast cancers reported in our institution. It is surprising the very low percent, compared with other published data, which report an incidence among all other breast cancers 10 -fold higher, respectively $1 \%$ [3,6]. The mean age was $61.34 \pm 11.198$, slightly lower than the age reported in other studies $[6,13]$ and the majority of cases were in a more advanced stage $(64.8 \%$ in stage III and IV) compared with other studies $[1,6,7,13,14]$, suggesting the lack of awareness about this cancer, in male population. Similarly to other published studies, the majority of cases $82.4 \%$ had the histology of intraductal carcinoma and more than $80 \%$ of the cases were estrogen and progesterone receptors (ER/PR) positive. None of our cases expressed triple negative 
histology. At the moment of this retrospective study 55.8\% patients were alive. Despite the low incidence of this pathology in male, the aggressiveness and severity of this malignancy needs special attention. The F18-FDG PET/CT hybrid imaging is an essential tool in primary diagnostic, staging, follow-up, and treatment response evaluation of malignant diseases, having an increasing role in the management of breast cancers. Compared with other studies, in our male breast cancer cohort, this method was not used for staging; the scanning was performed for restaging in $21.7 \%$ and the majority of scans were indicated because of the increase of the tumor markers (56.5\%). Just a few cases were referred for treatment response assessment (8.7\%) and similarly for follow-up, underling the lack of evidences, both in male and female breast cancers, for the utility of the method in the long-term monitoring of the disease. Current guidelines do not recommend intensive surveillance, including F18-FDG PET/CT, in asymptomatic breast cancer patients [15]. Our retrospective review of male patients with breast cancer demonstrates that F18-FDG PET/CT detects unsuspected distant metastases at a very high rate of $52.2 \%$, which is a rate above those published by Ulaner et al. [7] and other authors [6,9]; this fact might be explained by the advanced stages of our cases and also due to the complete lack of initial, baseline F18-FDG PET/CT imaging despite the advanced diseases. The rate of upstaging was lower than in other studies, occurring in 6 patients, mainly because our group consisted of patients in advanced stages; this fact suggested the role of F18-FDG PET/CT in the initial staging of male patients with breast cancer. The treatment plan for these patients needed to be changed from surgical management to systemic therapy without radical surgical procedure in 7 patients. The metastatic sites were confirmed through biopsy in 5 out of 12 patients with unsuspected metastases, in the rest of patients the tumor markers in dynamic evolution and the response to therapy were arguments for the positive diagnostic.

The number of 4 patients detected with synchronous tumors in F18-FDG PET/CT was a considerable high number. In this study we reported the occurrence of thyroid cancer and prostate cancer in association with breast cancer. In female, the association of thyroid-breast cancers as multiple primary malignancies is well studied, without having a clear scientific accepted explanation, except the more systematic screening. The interactions between thyroid and breast disorders are based on hormonal and cellular receptor mechanisms [16,17]. Thyroid cancer survivors also have been found to develop breast cancer early, have more estrogen and progesterone receptor positive tumors, and have a greater incidence of mixed invasive cancer [18]. Estrogen receptors have been found in thyroid tissue [19]. The histology of the breast cancer that develops after thyroid cancer is different than the general population, with a greater percentage of ER/PR-positive tumors [19]. Although indicated by many studies, an association between breast and thyroid cancer still remains controversial [20]. The high percentage of male patients with ER/PR positive might lead to the conclusion that male breast cancer patients need a careful examination and evaluation of the thyroid, in order to exclude the thyroid malignancy.

Regarding the association with prostate cancer a review of the existing literature shows that both prostate and breast cancers are typically hormone-dependent tumors and have similarities regarding etiology, epidemiology, and treatment approaches [21]. Alteration of estrogen to testosterone ratio is another possible explanation for the increased risk of hormone-sensitive cancers [22]. In this study we found breast cancer associated both with thyroid and prostate cancer. Despite the limited number of patients, we need to analyze the option of screening for thyroid and prostate cancers in male breast cancer patients.

The limitations of the study consist in the low number of patients in this cohort, but male breast cancer is an uncommon malignancy, and also, the number of synchronous malignancies detected in F18-FDG PET/CT was very limited; considering these facts, every study will contribute to the existing databases. The retrospective single-institution study is another limitation. Another limit of the study was the lack of baseline examinations, but F18-FDG PET/CT scans succeeded to correctly modify the staging and to determine the correct management of patients with multiple primary cancers. 


\section{Conclusions}

F18-FDG PET/CT is a valuable tool to provide information on extra-axillary lymph node involvement, distant metastases, and other occult primary cancers in the evaluation of male breast cancer. Baseline F18-FDG PET/CT has a substantial impact on initial staging and on clinical management in male breast patients and should be considered for use in newly diagnosed patients. Despite the rarity of the pathology, the occurrence of multiple primary cancers needs to be carefully evaluated, in this light, the role of hybrid imaging being essential.

Author Contributions: A.P., D.P.: conceptualization, data curation; D.P. and N.P.: formal analysis; C.C.: investigation; A.P., I.A., D.-S.M.: methodology; A.P., A.A.K., S.S.C.: project administration; D.P.: software; A.M.: supervision; C.C.: validation; S.S.C., A.P.: writing-original draft, A.P. All authors have read and agreed to the published version of the manuscript.

Funding: This paper was published under the frame of European Social Found, Human Capital Operational Programme 2014-2020, project no. POCU/380/6/13/125171.

Institutional Review Board Statement: The study protocol was approved by the Ethics Committee of the Institute of Oncology "Prof. Dr. I. Chiricuta" Cluj-Napoca, approval number 174/28.02.2020. The study was conducted according to the principles of the Declaration of Helsinki, the International Conference on Harmonization Guideline on Good Clinical Practice, the Romanian laws and regulations.

Informed Consent Statement: Informed consent was obtained from all subjects involved in the study.

Data Availability Statement: The data presented in this study are available on request from the corresponding author.

Conflicts of Interest: The authors declare no conflict of interest.

\section{References}

1. Macdonald, S.; Oncology, R.; General, M. Breast Cancer Breast Cancer. J. R. Soc. Med. 2016, 70, 515-517.

2. Ulaner, G.A.; Castillo, R.; Wills, J.; Gönen, M.; Goldman, D.A. (18)F-FDG-PET/CT for systemic staging of patients with newly diagnosed ER-positive and HER2-positive breast cancer. Eur. J. Nucl. Med. Mol. Imaging 2017, 44, 1420-1427. [CrossRef] [PubMed]

3. Groheux, D. FDG-PET/CT for systemic staging of patients with newly diagnosed breast cancer. Eur. J. Nucl. Med. Mol. Imaging 2017, 44, 1417-1419. [CrossRef] [PubMed]

4. Hyland, C.J.; Varghese, F.; Yau, C.; Beckwith, H.; Khoury, K.; Varnado, W.; Hirst, G.L.; Flavell, R.R.; Chien, A.J.; Yee, D.; et al. Use of 18F-FDG PET/CT as an Initial Staging Procedure for Stage II-III Breast Cancer: A Multicenter Value Analysis. J. Natl. Compr. Cancer Netw. 2020, 18, 1510-1517. [CrossRef]

5. Ko, H.; Baghdadi, Y.; Love, C.; Sparano, J.A. Clinical utility of 18F-FDG-PET/CT in staging localized breast cancer prior to initiating preoperative systemic therapy. J. Clin. Oncol. 2020, 38, 563. [CrossRef]

6. $\quad$ Evangelista, L.; Bertagna, F.; Bertoli, M.; Stela, T.; Saladini, G.; Giubbini, R. Diagnostic and Prognostic Value of 18F-FDG PET/CT in Male Breast Cancer: Results From a Bicentric Population. Curr. Radiopharm. 2016, 9, 169-177. [CrossRef] [PubMed]

7. Ulaner, G.A.; Juarez, J.; Riedl, C.C.; Goldman, D.A. (18)F-FDG PET/CT for Systemic Staging of Newly Diagnosed Breast Cancer in Men. J. Nucl. Med. 2019, 60, 472-477. [CrossRef]

8. Groheux, D.; Hindié, E.; Marty, M.; Espié, M.; Rubello, D.; Vercellino, L.; Bousquet, G.; Ohnona, J.; Toubert, M.-E.; Merlet, P.; et al. ${ }^{18}$ F-FDG-PET/CT in staging, restaging, and treatment response assessment of male breast cancer. Eur. J. Radiol. 2014, 83, 1925-1933. [CrossRef]

9. McEachen, J.C.; Kuo, P.H. Male primary breast cancer found on FDG-PET/CT. Clin. Nucl. Med. 2008, 33, 630-632. [CrossRef]

10. Sarma, M.; Borde, C.; Subramanyam, P.; Shanmuga Sundaram, P. Random synchronous malignancy in male breast: A case report. J. Breast Cancer 2013, 16, 442-446. [CrossRef]

11. Vadi, S.K.; Mittal, B.R.; Sood, A.; Singh, G.; Bal, A.; Parihar, A.S.; Bhattacharya, A.; Basher, R.K.; Kapoor, R. Diagnostic and prognostic value of 18F-FDG PET/CT imaging in suspected recurrence of male breast cancer. Nucl. Med. Commun. 2019, 40, 63-72. [CrossRef]

12. Boellaard, R.; Delgado-Bolton, R.; Oyen, W.J.G.; Giammarile, F.; Tatsch, K.; Eschner, W.; Verzijlbergen, F.J.; Barrington, S.F.; Pike, L.C.; Weber, W.A.; et al. FDG PET/CT: EANM procedure guidelines for tumour imaging: Version 2.0. Eur. J. Nucl. Med. Mol. Imaging 2015, 42, 328-354. [CrossRef] [PubMed]

13. Srour, M.K.; Amersi, F.; Mirocha, J.; Giuliano, A.E.; Chung, A. Male Breast Cancer: 13-Year Single Institution Experience. Am. Surg. 2020, 86, 1345-1350. [CrossRef] [PubMed]

14. Swamy, N.; Rohilla, M.; Raichandani, S.; Bryant-Smith, G. Epidemiology of male breast diseases: A 10-year institutional review. Clin. Imaging 2020, 72, 142-150. [CrossRef] [PubMed] 
15. Bick, U.; Helbich, T.H. Follow-Up of Patients with Breast Cancer: Imaging of Local Recurrence and Distant Metastases; Hodler, J., Kubik-Huch, R.A., von Schulthess, G.K., Eds.; Springer: Cham, Switzerland, 2019; pp. 167-178. ISBN 978-3-030-11148-9.

16. Giani, C.; Fierabracci, P.; Bonacci, R.; Gigliotti, A.; Campani, D.; De Negri, F.; Cecchetti, D.; Martino, E.; Pinchera, A. Relationship between breast cancer and thyroid disease: Relevance of autoimmune thyroid disorders in breast malignancy. J. Clin. Endocrinol. Metab. 1996, 81, 990-994. [PubMed]

17. Kuo, J.H.; Chabot, J.A.; Lee, J.A. Breast cancer in thyroid cancer survivors: An analysis of the Surveillance, Epidemiology, and End Results-9 database. Surgery 2016, 159, 23-29. [CrossRef]

18. Bhatia, S.; Sklar, C. Second cancers in survivors of childhood cancer. Nat. Rev. Cancer 2002, 2, 124-132. [CrossRef]

19. Kawabata, W.; Suzuki, T.; Moriya, T.; Fujimori, K.; Naganuma, H.; Inoue, S.; Kinouchi, Y.; Kameyama, K.; Takami, H.; Shimosegawa, T.; et al. Estrogen receptors (alpha and beta) and 17beta-hydroxysteroid dehydrogenase type 1 and 2 in thyroid disorders: Possible in situ estrogen synthesis and actions. Mod. Pathol. 2003, 16, 437-444. [CrossRef] [PubMed]

20. Arer, İ.M.; Yabanoğlu, H.; Kuş, M.; Akdur, A.; Avcl, T. Retrospective Analysis of Patients with Synchronous Primary Breast and Thyroid Carcinoma. Eur. J. Breast Health 2018, 14, 80-84. [CrossRef]

21. Moosavi, L.; Kim, P.; Uche, A.; Cobos, E. A Synchronous Diagnosis of Metastatic Male Breast Cancer and Prostate Cancer. J. Investig. Med. High Impact Case Rep. 2019, 7, 2324709619847230. [CrossRef]

22. Rudlowski, C. Male Breast Cancer. Breast Care (Bassel) 2008, 3, 183-189. [CrossRef] [PubMed] 